ThE JOURNAL OF CHEMICAL PHYSICS VOLUME 16, NUMBER 9 SEPTEMBR, 1948

\title{
Note on an Equation of State for Linear Macromolecules in Monolayers*
}

\author{
S. J. SINGER** \\ Gates and Crellin Laboratories, California Institute of Technology, Pasadena, California
}

(Received April 15, 1948)

\begin{abstract}
An equation of state for monolayers of large threadlike molecules based on the highly idealized quasi-lattice model is presented, and applied with fair success to the small number of experimental data available. Although protein molecules in monolayers may be highly organized structures, the theory can be applied to them at moderate film pressures without requiring unreasonable values for their molecular parameters. By analogy with the threedimensional case, the theory requires improvement in order to apply to the low pressure regions, where experimental differences between protein and polymer films should become more marked.
\end{abstract}

$I^{\mathrm{N}}$ recent years considerable interest has centered about the experimental study of monomolecular films of large molecules. The structure of cellulose and its derivatives, ${ }^{1}$ and of synthetic high polymers $^{2-4}$ have been investigated by this technique. A greater number of papers have been published dealing with monolayers of protein molecules, in the determination of their size and structure ${ }^{5-7}$ and in the nature of their reactions. ${ }^{8}$ In this paper we will present an equation of state for macromolecular monolayers derived on the basis of a highly idealized model, which should have significance for the films of synthetic polymers, but whose application to those of proteins is rendered uncertain by the nature of the model.

The investigations cited show that in true monomolecular films, polymer and protein molecules alike lie flat and unfolded at the interphase. It is interesting that films of both polymers and proteins are highly compressible, and exhibit comparable force-area relations, though in threedimensional solution the protein is generally much more rigid and compact than the polymer molecule. However, while the latter might be

* Contribution No. 1198 from the Gates and Crellin Laboratories of the California Institute of Technology.

** Abbott Research Fellow, 1947-48.

${ }^{1}$ N. K. Adam, Trans. Faraday Soc. 29, 90 (1933).

${ }^{2}$ W. D. Harkins, E. F. Carman, and H. E. Reis, Jr., J. Chem. Phys. 3, 692 (1935).

${ }^{3}$ F. T. Wall and M. Zelikoff, J. Am. Chem. Soc. 68, 726 (1946).

${ }^{4}$ D. J. Crisp, J. Coll. Sci. 1, 49, 161 (1946).

${ }^{5}$ I. Langmuir and V. J. Schaefer, Chem. Rev. 24, 181 (1939).

${ }^{6}$ H. B. Bull, J. Am. Chem. Soc. 67, 4, 8, 10 (1945) ; 68, 745 (1946).

${ }^{7} \mathrm{~J}$. Gaustella, J. chim. phys. 42, 71 (1945).

8 A. Rothen, J. Biol. Chem. 168, 75 (1947). expected to exist as a more or less randomly coiled thread on the surface, loosely anchored to the aqueous substrate by polar groups distributed along the thread, the protein molecule may unfold into a more or less well knit sheet on the surface, retaining a considerable degree of internal hydrogen bonding. If this is so, application of this random-coil theory to protein films becomes ambiguous. Nevertheless, as will be shown below, upon comparing theory with experiment for protein films, no startling discrepancies arise.

Our model of the surface at the interphase is a two-dimensional quasi-lattice in which the linear macromolecule may move about, taking on a great number of energetically equivalent configurations, and our calculations are exactly analogous to those of Huggins ${ }^{9}$ and Flory ${ }^{10}$ describing the thermodynamic behavior of threedimensional solutions of polymers. The total surface of area $\sigma$ is composed of $N_{0}$ cells of area $\sigma_{0}$ each. In this first approximation, we neglect energies of interaction between solvent and polymer molecules, whether situated in or out of the interphase, and it is therefore immaterial whether we consider the surface occupied by solvent plus polymer molecules, or holes plus polymer molecules, or by all three. Consider that. there are, finally, $N$ large molecules on the surface, each with $x$ identical submolecules (monomer units), and $\boldsymbol{n}$ remaining sites for holes or solvent molecules, the areas occupied by a single submolecule and a solvent molecule being

${ }^{9}$ (a) M. L. Huggins, J. Phys. Chem. 46, 151 (1942);

(b) M. L. Huggins, Ann. N. Y. Acad. Sci. 43, 1 (1942).

${ }_{10}$ P. J. Flory, J. Chem. Phys. 10, 51 (1942). 
the same, $\sigma_{0}$. We count the number of different ways we can place the polymer submolecules in the lattice, adding one polymer molecule at a time. Proceeding by the method of Huggins, ${ }^{9 b}$ we find that total number of different configurations, $\Phi$, to be :

$$
\Phi=\frac{\prod \nu_{i, j}}{N ! \beta^{N}} ; \quad \Pi \nu_{i, j}=\prod_{i=1}^{N} \frac{N_{0} z y^{x-2}\left(1-f_{i, j}\right)^{x}}{\left(1-\frac{2(x-1)}{x z} f_{i, j}\right)^{x-1}} .
$$

Here $\nu_{i, j}$ is the number of sites available to the $j$ th submolecule of the $i$ th polymer molecule; $z$ is the coordination number of the lattice; $y=\nu_{1,3} \simeq_{z}-1 ; f_{i, j}$ is the fraction of sites already occupied when the $j$ th submolecule of the $i$ th polymer molecule is added to the lattice, and $\beta$ is the symmetry number of the polymer chain. For $x \geqslant 10$, we can write

$$
f_{i j}=f_{0}+\frac{(i-1) x\left(1-f_{0}\right)}{N_{0}},
$$

where $f_{0}$ is some small constant. For $x<10, f_{0} \sim 0$. We take logarithms of both sides of Eq. (1), replace summations by integrals, factorials by their Stirling approximations, and utilizing Eq. (2), arrive at an equation for the change in the surface entropy, $\Delta S_{s}$, produced by placing the $N$ polymer molecules in the lattice:

$$
\begin{array}{r}
\Delta S_{s}=k \ln \Phi=k\left\{N \ln \left(\frac{z y^{x-2}}{\beta}\right)+(N+n) \ln N_{0}\right. \\
-N \ln N-n \ln n-2 N+x N \ln \left(1-f_{0}\right) \\
-(x-1) N \ln \left[1-\frac{2(x-1) f_{0}}{x z^{\prime}}\right] \\
-(x-1)\left[\frac{z^{\prime} n}{2 x}+\left(\frac{z^{\prime}}{2}-1\right) N\right] \\
\left.\quad \times \ln \left[\frac{N_{0}}{n+\left(1-2 / z^{\prime}\right) x N}\right]\right\} .
\end{array}
$$

In this equation

$$
z^{\prime}=\frac{z x}{(x-1)\left(1-f_{0}\right)}-\frac{2 f_{0}}{\left(1-f_{0}\right)}
$$

for practical purposes, a constant.
The film pressure, $\pi$, is given by $\pi=\gamma_{w}-\gamma_{F}$, the surface tension of water minus the surface tension of the water covered with the film. $\pi=\left(\partial \Delta F_{s} / \partial \sigma\right)_{N, T}=-T\left(\partial \Delta S_{s} / \partial \sigma\right)_{N, T}$, where $\Delta F_{s}$ is the surface free energy change, since we are neglecting the variation in the surface enthalpy. Substituting into Eq. (3), the relations $n=N_{0}$ $-x N$, and $N_{0}=\sigma / \sigma_{0}$, and performing the indicated operations, we find

$$
\begin{aligned}
\pi=\frac{k T}{\sigma_{0}}\left\{\begin{array}{c}
(x-1) z^{\prime} \\
2 x
\end{array}\right) & \ln \left(1-\frac{2 x N \sigma_{0}}{\sigma z^{\prime}}\right) \\
& \left.-\ln \left(1-\frac{x N \sigma_{0}}{\sigma}\right)\right\} .
\end{aligned}
$$

For $\left(x N \sigma_{0} / \sigma\right) \ll 1$, this reduces to the perfect gas law, $\pi=N k T / \sigma$. If we let $k T / \sigma_{0}=\pi_{0}, \sigma / N=A$, the observed area per molecule, and $x \sigma_{0}=A_{0}$, the condensed area per molecule, Eq. (4) is modified to a reduced equation of state:

$$
\frac{\pi}{\pi_{0}}=\left[\frac{(x-1) z^{\prime}}{2 x} \ln \left(1-\frac{2}{z^{\prime}} \frac{A_{0}}{A}\right)-\ln \left(1-\frac{A_{0}}{A}\right)\right] \text {. }
$$

Aside from the imperfections of the quasilattice model, two approximations are seriously to be considered: (a) the neglect of energies of interactions, and (b) the assumption that, on adding a polymer molecule to the lattice, the segments of those polymer molecules already placed in the lattice are randomly situated among occupied sites. Since no extensive investigations have as yet been made of the temperature dependence of the pressure-area curves of monolayers of macromolecules, the effect of the first of these approximations is not known. It will be better satisfied in dilute than in concentrated surface solutions. Attempts have been made to improve the second approximation, ${ }^{11}$ but no satisfactory theory covering the entire concentration range has yet been obtained. In very dilute solutions, deviations of theory from experiment will arise from this source. In addition, one might think it possible that the molecules occupy more than one molecular thickness in the third dimension, instead of being restricted to coil in two dimensions only, but this effect would provide for greater compressibility of the

"I P. J. Flory, J. Chem. Phys. 13, 453 (1945). 


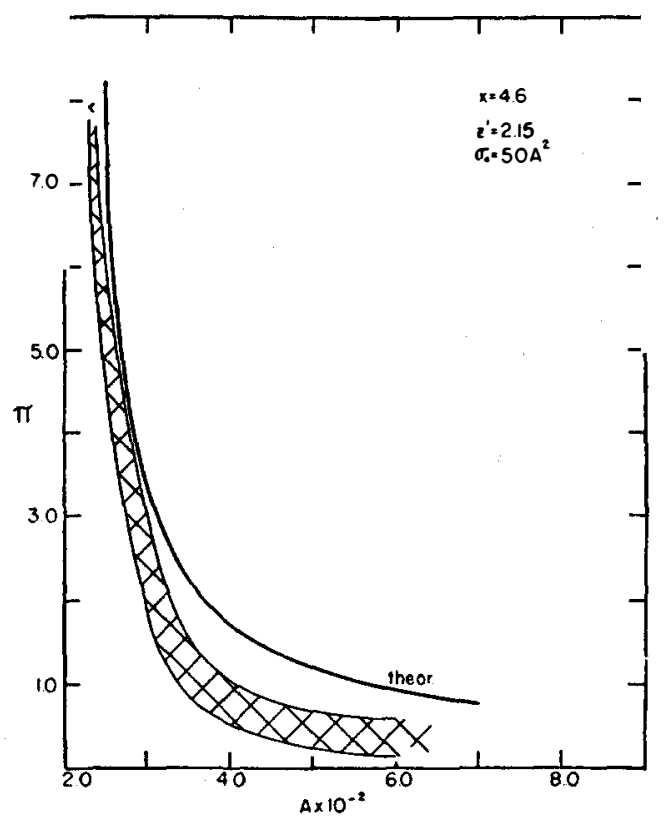

Fig. 1.

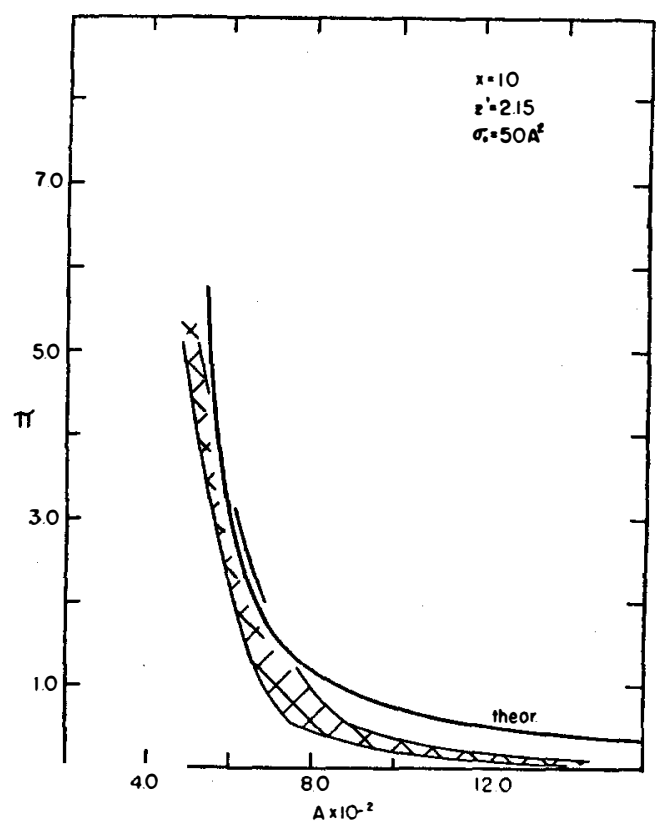

Fig. 2.

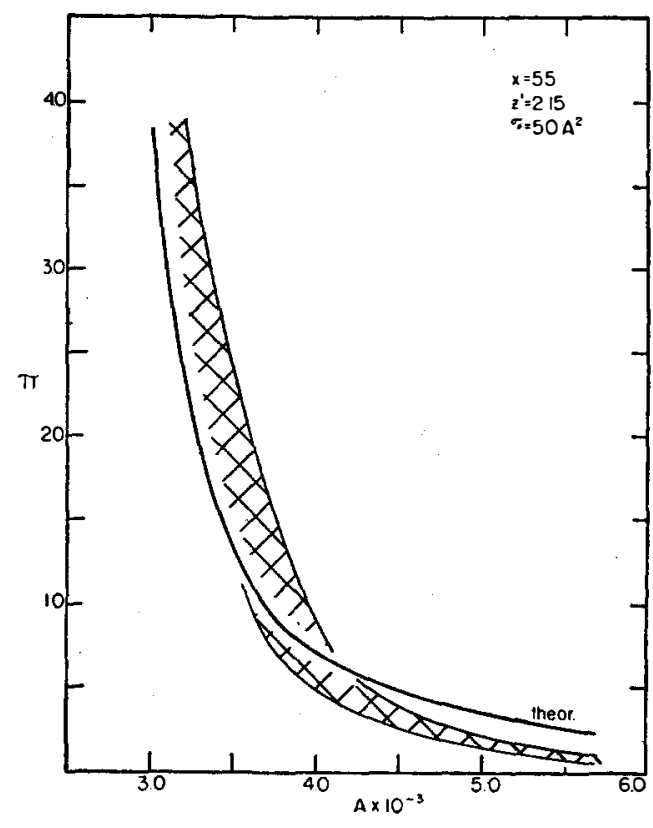

Fig. 3.

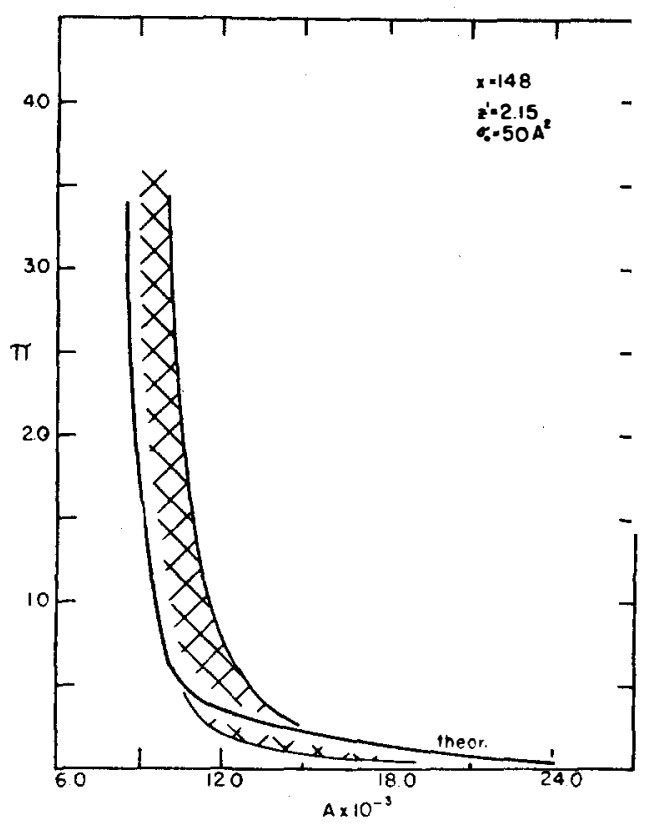

Fig. 4.

FIgs. 1-4. Comparison of theoretical and experimental force-area relations for $\omega$-hydroxy decanoic acid polymers. ${ }^{2}$ Cross-hatched areas represent the variations in the experimental curves in going from $0.01 N \mathrm{NaOH}$ to $0.01 N \mathrm{HCl}$ as substrate.

films, whereas the theory as it stands already appears to err in that direction.

We may now proceed to compare the theory with experiment qualitatively. Equation $\left(4^{\prime}\right)$ permits one to evaluate the effect of molecular weight on pressure-area relations for homologous series of polymers. For a variety of polymer series, it has been found ${ }^{1,2,4}$ that at moderate pressures the area per molecule is practically a linear function of molecular weight, or, i.e., that 
the area per unit of polymer is nearly independent of molecular weight. From Eq. $\left(4^{\prime}\right)$ it is clear that for $x>10$, and for moderate film pressure, the relation between $\pi / \pi_{0}$ and $A / A_{0}$ is practically independent of molecular weight, in accord with the experimental fact. Moreover, the small dependence of the reduced pressure-area relation on molecular weight predicted by Eq. $\left(4^{\prime}\right)$ is such that the curves for lower molecular weight species lie slightly above those of their higher molecular weight homologues. This has been found to be so by Adams, ${ }^{1}$ with some nitro. cellulose spread on $2 \mathrm{~N} \mathrm{NaOH}$, and by Harkins et al. ${ }^{2}$ with a series of $\omega$-hydroxy decanoic acid polymers spread on dilute acid and base.

Quantitative comparison of theory with experiment requires knowledge of three parameters, $z^{\prime}, x$, and $\sigma_{0}$. Of the studies on synthetic polymers, that of Harkins et al. is the only one amenable to such a calculation. The number average molecular weights of the various $\omega$-hydroxy decanoic acid polymers investigated range from 780 to $25,200, x$ varying from 4.6 to 148 . A reasonable value of $\sigma_{0}$ for the $\left(\mathrm{CH}_{2}\right)_{9} \mathrm{COO}$ unit is about $50 \mathrm{~A}^{2}$, while for $z^{\prime}$, a value between 2 and 4 is to be expected. In Figs. 1 to 4 are plotted the theoretical and experimental curves for four of these polymers, using $z^{\prime}=2.15$ and $\sigma_{0}=50 \mathrm{~A}^{2}$. The cross-hatched areas represent the variation in the pressure-area relations caused by changing the $p \mathrm{H}$ of the substrate. Concerning the fraction of lowest molecular weight, the value of $x=4.6$ is below that required by the use of certain approximations in the theory, but even so, the same values of the parameters yield the proper order of magnitude for the relation. However, the data seem to indicate that irrespective of molecular weight a lower value of $z^{\prime}$ is required in the low pressure region than in the high.

In attempting to interpret the data obtained with protein monolayers, certain experimental difficulties should first be realized. Various investigators do not agree on their experimental results, possibly because of difficulties in attaining equilibrium, or from the considerably increased solubility of some spread proteins upon increase of film pressure, or for a variety of other reasons.

Moreover, as we have mentioned above, the structure of the protein molecule in a film may be considerably different from a random coil because of intramolecular hydrogen bonding. Further complications might arise from the possibility of the side chains on the polypeptide backbone changing their orientation on compression of the film. Qualitatively, however, the pressure-area curves for proteins so far investigated resemble one another considerably, and are quite similar to those observed with polymers. In particular, there is no indication that phase changes of the kind. appearing in films of low molecular weight compounds ${ }^{12}$ occur with protein films.

As an example, we have attempted to fit the data of Bull ${ }^{6}$ on egg albumin monolayers spread on 35 percent ammonium sulfate solution. Using $z^{\prime}=2.10$ and $\sigma_{0}=14.5 \mathrm{~A}^{2}$, we obtain the curve shown in Fig. 5 for the high pressure region. The value of $\sigma_{0}=14.5 \mathrm{~A}^{2}$ is slightly lower than that found by Bull, $15.1-15.6 \mathrm{~A}^{2}$, from the area corresponding to the minimum in the compressibility versus area relation (compressibility = $-\partial A / A \partial \pi)$, and by Palmer and Galvin, ${ }^{13} 15.4 \mathrm{~A}^{2}$,

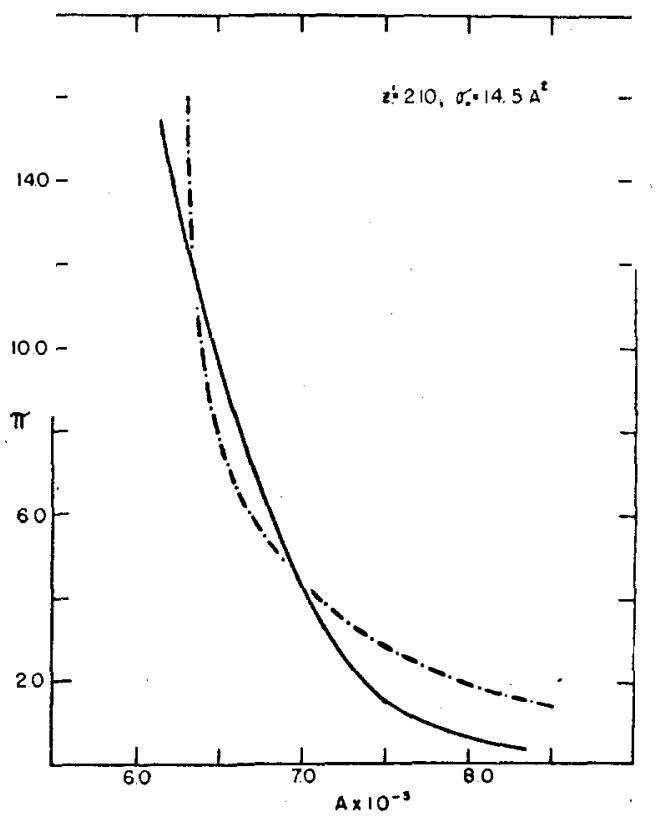

Fig. 5. Comparison of theoretical and experimental force-area relations in the high pressure region for egg albumin ${ }^{6}$ spread on 35 percent $\left(\mathrm{NH}_{4}\right)_{2} \mathrm{SO}_{4}$. The solid line represents the experimental curve.

${ }^{12}$ W. D. Harkins, in J. Alexander, Colloid Chemistry (Reinhold Publishing Corporation, New York, 1944), Vol. V, p. 22.

${ }_{13}$ K. J. Palmer and J. A. Galvin, J. Am. Chem. Soc. 65, 2187 (1943). 


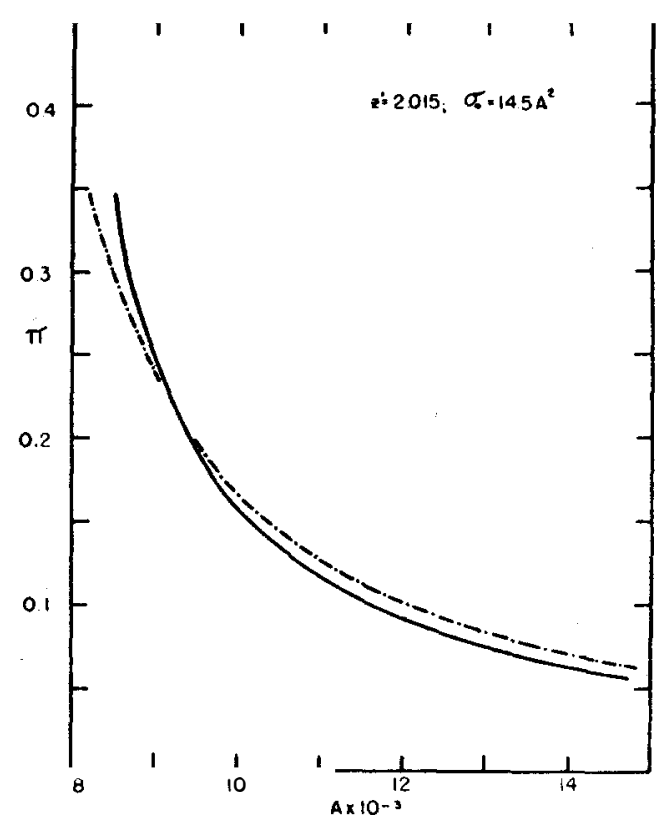

FIg. 6. Same as Fig. 5 in the low pressure region.

from $x$-ray data on fibers of egg albumin. Bull's value, however, is expected to be somewhat larger than ours. A minimum in the film compressibility is an indication of film collapse, and the area per molecule at collapse will be larger than $A_{0}$. The correspondence of the theoretical and experimental curves is not as good as with the polymers. To fit the data in the low pressure region, below 0.5 dynes $\mathrm{cm}^{-1}$, a lower value of $z^{\prime}, 2.015$, is required, as is shown in Fig. 6. The need for a revised value of $z^{\prime}$ also appears to be the case with the $\omega$-hydroxy decanoic acid polymers. A similar effect is noted in the case of three-dimensional polymer solutions, ${ }^{11}$ a smaller coordination number being required in dilute than in concentrated solutions. Probably, then, a similar cause is responsible, namely, the fact that in dilute solutions the approximation of randomly ,occupied sites does not hold as well as in more concentrated ones. That is, the distribution of occupied sites becomes markedly discontinuous when the molecules are, on the average, far from each other. If necessary, an approximate theory for the low pressure, or "gaseous film," region corresponding to that of Flory ${ }^{11}$ for dilute three-dimensional solutions could be readily developed. The lack of data in the low pressure regions, however, makes this hardly worth while at present. It should be realized, however, that if experimental problems can be overcome, the low pressure region will yield valuable information concerning not only the molecular weight, ${ }^{6}$ but particularly the structure of macromolecular films. If protein molecules form fairly well knit structures on the interphase as compared to those of polymers, it is in this region that marked differences in the pressure-area relations will appear, the proteins behaving more nearly as ideal twodimensional gases than polymers at comparable low pressures.

The author wishes to express his sincere gratitude to Professor J. G. Kirkwood for his many helpful suggestions. 